\title{
Incidencia de los defectos del tubo neural en el Instituto Nacional Materno Perinatal de Lima
}

\author{
CAROLINA TARQUI-MAMANI ${ }^{(1,2)}$, HERNÁN SANABRIA ${ }^{(1,2)}$, NELLY LAM $^{(3,4)}$ y JUAN ARIAS ${ }^{(4)}$ \\ RESUMEN
}

Objetivo: Determinar la tendencia de la tasa de incidencia hospitalaria de DTN y sus tipos durante el periodo de prefortificación (2001-2005) en el INMP. Material y método: Se realizó un estudio observacional, descriptivo, retrospectivo y de corte transversal. Se revisaron historias clínicas de recién nacidos y sus madres entre 2001-2005. El universo muestral fue 93.863 RN, de los cuales 128 RN tuvieron DTN. El análisis univariado incluyó promedio, desviación estándar, determinación de frecuencias absolutas y relativas y la comparación entre los datos de DTN, según los diferentes años, se realizó mediante una prueba de Prais Winsten. Se calculó el IC 95\% para la incidencia global y los tipos de DTN a través del Test de Poisson. Resultados: Los DTN fluctuaron entre 18 y 32 casos por año, correspondiendo una tasa hospitalaria global de 13,6 por 10000 RN (IC 95\%: 11,38;16,21) entre el 2000-2005. La incidencia de espina bifida fue 8,3x 10000 (IC 95\%: 6,57;10,37), seguida de anencefalia con 5,1 10000 (IC 95\%: 3,77;6,78) y 0,2 x 10000 (IC 95\%: 0,03; 0,77) para encefalocele. Finalmente, la tendencia de DTN entre el 2001 al 2005 fue ascendente no significativa $(p=0,951)$. Conclusión: El promedio de la TGI de DTN en el periodo 2001-2005 fue de 13,6 por 10,000 RN (IC: 11,38;16,21), siendo su tendencia ascendente no significativa. El mielomeningocele tuvo la más alta tasa de incidencia en relación a los otros tipos de DTN.

Palabras clave: tubo neural, malformación, ácido fólico, fortificación, incidencia.

\section{ABSTRACT \\ INCIDENCE OF NEURAL TUBE DEFECTS IN THE NATIONAL MATERNAL-PERINATAL INSTITUTE OF LIMA}

Objective: Determine the hospital incidence rate trends for neural tube defects (NTDs) and their types during the pre-fortification period (2001-2005) at the National Maternal-Perinatal Institute of Lima (INMP). Material and method: An observational, descriptive, retrospective, cross sectional study was carried out. Medical histories of newborns and their mothers between 2001-2005 were reviewed. The sampling universe was 93.863 newborns, of which 128 had NTDs. The univariate analysis included the average, standard deviation, and absolute and relevant frequency determination. The comparison between the data for different years was carried out through a Prais Winsten test. The CI of 95\% for the global incidence and the types of NTD was calculated through the Poisson Test. Results: NTDs fluctuated between 18 and 32 cases per year, which corresponds to the global hospital rate of 13.6 per 10,000 newborns (CI 95\%: 11.38;16.21) between 2000-2005. The incidence of spina bifida was $8.3 \times 10,000$ (CI 95\%: 6.57;10.37), followed

(1) Departamento de Medicina Preventiva y Salud Pública. Facultad de Medicina San Fernando de la Universidad Nacional Mayor de San Marcos. José María Plaza No 458. Dpto. 304. Jesús María. Lima. Perú. carobtm@hotmail.com.

(2) Instituto Nacional de Salud. Ministerio de Salud. Lima. Perú.

(3) Departamento de Gíneco-obstetricia. Facultad de Medicina San Fernando de la Universidad Nacional Mayor de San Marcos. Lima. Perú.

(4) Instituto Nacional Materno Perinatal. Ministerio de Salud. Lima. Perú. 
by anencephaly with $5.1 \times 10,000$ (CI 95\%: 3.77;6.78) and $0.2 \times 10,000$ (CI 95\%: 0.03; 0.77) for encephalocele. Finally, the tendency of NTDs between 2001 to 2005 showed a not significant increase ( $p=0.951)$. Conclusion: The average NTD global incidence rate in the period of 20012005 was 13.6 per 10,000 newborns (CI: 11.38;16.21), showing a not significant rising trend. Myelomeningocele had the highest incidence rate in relation to the other types of NTD.

Keywords: neural tube, malformation, folic acid, fortification, incidence.

\section{INTRODUCCIÓN}

Los defectos del tubo neural (DTN) en sus diferentes formas, anencefalia, espina bífida y encefalocele, se producen durante la embriogénesis temprana ${ }^{1,2}$ al no cerrarse el tubo neural durante el primer mes de embarazo. La espina bífida es el defecto que se produce en el $50 \%$ de los casos de DTN, la anencefalia en el $40 \%$ y el encefalocele en el $10 \%{ }^{3}$. En ello incide la interacción de factores ambientales, nutricionales, genéticos y étnicos, también se reconocen factores causales desconocidos ${ }^{4}$. $\mathrm{La}$ anencefalia y el encefalocele son lesiones incompatibles con la vida, a diferencia de la espina bífida que es fatal, pero involucra un alto grado de deficiencia y discapacidad en las personas afectadas.

Las formas de DTN son: Espina bífida: es el DTN más frecuente ${ }^{3} \mathrm{y}$ de mejor pronóstico de vida. Se reconocen 3 formas de espina bífida: oculta, meningocele y el mielomeningocele. Anencefalia: es el DTN más severo ${ }^{3}$ y es fatal, el recién nacido - RN- tiene un cerebro poco desarrollado y cráneo incompleto. Encefalocele: es el DTN más raro ${ }^{3}$, que resulta de una abertura anormal en el cráneo por la que el cerebro protruye. Suele acompañarse de otras deformidades del cráneo y de la cara y se manifiesta con hidrocefalia, cuadriplejía espástica, retardo en el desarrollo, problemas de visión, retardo mental y de crecimiento así como de convulsiones. La mayoría de $\mathrm{RN}$ no sobrevive o es muy retardado.

En relación a mortalidad, el $50 \%$ de los afectados por DTN fallece en el primer año de vida y los que sobreviven tienen discapacidades físicas y/o mentales que requieren de rehabilitación larga y costosa ${ }^{5}$. En México ${ }^{6}$, durante el periodo 1980-1997, se encontraron
5,8 por 10.000 nacidos vivos de tasa bruta de mortalidad por DTN, siendo la anencefalia el defecto más frecuente $(37,7 \%)$, seguida de la espina bífida sin hidrocefalia $(31,6 \%)$. Anencefalia y encefalocele son incompatibles con la vida.

La incidencia mundial de los DTN oscila entre 1 a 8 casos por cada 10000 nacidos vivos $(\mathrm{NV})$. Este defecto predomina en la raza caucásica y en poblaciones de niveles socioeconómicos bajos ${ }^{3}$. En el 2003 se notificaron 350.000 nuevos casos de DTN a nivel mundial. En América Latina existe escasa información al respecto, salvo el registro hospitalario sobre DTN del Estudio Colaborativo Latinoamericano de Malformaciones Congénitas (Eclamc). En 1995, el Eclamc dio a conocer las siguientes tasas de incidencia por 10.000 nacidos vivos: 7,6 para la anencefalia, 9,4 para la espina bífida y 1,6 para el encefalocele ${ }^{7}$. En 1989, en un solo hospital de Colombia se encontró una tasa general de DTN de 13 por 10.000 nacidos vivos $^{8}$; en 1995, el Registro y Vigilancia Epidemiológica de Malformaciones Congénitas Externas (Ryvemce) de México notificó 16,4; 8,9 y 3,1 por 10.000 nacidos vivos para la anencefalia, espina bífida y encefalocele respectivamente ${ }^{8}$. En Chile, el registro de Eclamc que cubre el $7 \%$ de los nacimientos notificó una tasa de 17 por 10.000 nacidos vivos ${ }^{7}$, confirmado luego por su sistema hospitalario de vigilancia de nacimientos en 1999'. En Costa Rica, se ha reportado una tasa de 9,7 de DTN por 10.000 nacimientos en el año $2000^{10}$ en la etapa de prefortificación de la harina con ácido fólico.

Durante el embarazo las necesidades maternas de folatos aumentan debido a la síntesis de ácidos nucleicos y proteínas durante la embriogénesis, velocidad de crecimiento y desarrollo fetal de los primeros meses de la 
gestación ${ }^{11}$. Las raciones dietéticas recomendadas de la National Academy of Sciences, Food and Nutrition Board, mencionan que la cantidad diaria de folatos que una mujer adulta necesita es de $200 \mu \mathrm{g} / \mathrm{día}$, mientras que la gestante requiere $400 \mu \mathrm{g} / \mathrm{dí}^{1} 2$. Múltiples estudios han demostrado que si la mujer consume ácido fólico a dosis adecuadas durante el periodo periconcepcional, disminuye el riesgo de ocurrencia y recurrencia de hijos con DTN. En Chile, después de iniciada la fortificación de la harina de trigo con ácido fólico en el año 2000 , la tasa de DTN ha disminuido a 10 por 10.000 nacimientos ${ }^{9}$. En Costa Rica ${ }^{10}$ la tasa de DTN bajó a 6,3 por 10.000 , por lo que se acepta que es posible disminuir los DTN entre un $50 \mathrm{y}$ $70 \%$.

$\mathrm{Al}$ reconocerse que el ácido fólico reduce la tasa de DTN, varios países emitieron normas legales fortificando la harina de trigo con ácido fólico y otros micronutrientes ${ }^{13-15}$. En el Perú se normó la fortificación de la harina de trigo mediante el Decreto Supremo 008-2005-SA aprobado por el Ministerio de Salud (Minsa). Esta norma estableció que toda la harina de trigo de producción nacional o importada debería estar fortificada a partir del 4 de agosto del 2005 de manera obligatoria según Ley $\mathrm{N}^{\mathrm{o}} 28.314$. Esta ley dispuso la fortificación de la harina de trigo con hierro $(55 \mathrm{mg} / \mathrm{kg})$, niacina $(48 \mathrm{mg} / \mathrm{kg})$, ácido fólico $(1.2 \mathrm{mg} / \mathrm{kg})$, vitamina B1 $(5 \mathrm{mg} / \mathrm{kg})$, y B2 $(4 \mathrm{mg} / \mathrm{kg})$. Sin embargo, la referida ley y su correspondiente implementación no se basaron en estudios científicos ${ }^{16}$, es decir, estudios de línea de base que hubieran permitido cuantificar el impacto de la fortificación de la harina de trigo. Basados en esta falta de información, en el Perú se propuso determinar la tasa de incidencia hospitalaria (TIH) de DTN y su tendencia durante el periodo prefortificación (2001-2005), así como describir algunas características sociodemográficas y de salud de la madre y del RN con DTN. El estudio se realizó en el INMP de Lima porque es el establecimiento de salud que atiende el mayor número de partos a nivel nacional, alrededor de 30 a 50 por día, aproximadamente 18.000 nacimientos por año; el INMP atiende además a gestantes de alto riesgo procedentes de todos los distritos de Lima y algunas de departamentos del interior.

\section{MATERIAL Y MÉTODO}

El estudio fue observacional, descriptivo, retrospectivo y transversal; la recolección de los datos se realizó durante el 2007 en el INMP previa autorización. Se incluyó al total de recién nacidos entre los años 2001 al 2005. El universo muestral estuvo constituido por 93.863 recién nacidos.

El punto de partida fue la identificación de todos los RN con diagnóstico de DTN en los registros o historias clínicas de los RN o madre durante el periodo de estudio. Se definió como caso de DTN a todo $\mathrm{RN}$ con la presencia de alguna forma de la malformación clasificada de acuerdo a su tipo: espina bífida, anencefalia y encefalocele, cuyo diagnóstico fue dado por el especialista que atendió al RN. La TIH de DTN se refiere a casos nuevos de RN con DTN entre el total de $\mathrm{RN}$ por 10.000. La tendencia se refiere

Tabla 1. Tasa de Incidencia DTN x 10000 (2001- 2005). Estudio en el INMP de Lima, Perú.

\begin{tabular}{|c|c|c|c|c|}
\hline \multirow{2}{*}{ DTN } & FORMAS & TOTAL & TASAS & IC 95\% (Poisson) \\
\hline \multirow{3}{*}{ Espina bífida } & Espina bífida & 7 & 0,8 & 0,$30 ; 1,54$ \\
\cline { 2 - 5 } & Mielomeningocele & 69 & 7,4 & 5,$72 ; 9,30$ \\
\cline { 2 - 5 } & Meningocele & 2 & 0,2 & 0,$03 ; 0,77$ \\
\hline \multicolumn{2}{|c|}{ Espina } & 78 & 8,3 & 6,$57 ; 10,37$ \\
\hline Encefalocele & 2 & 0,2 & 0,$03 ; 0,77$ \\
\hline \multicolumn{2}{|c|}{ Anencefalia } & 48 & 5,1 & 3,$77 ; 6,78$ \\
\hline
\end{tabular}


a la evolución de la TIH de DTN.

Se utilizó una ficha de datos ad hoc en la que consignó la evaluación clínica del RN: edad al nacer, sexo, peso, apgar, diagnóstico clínico, fecha y hora de nacimiento, tipo de parto y control prenatal entre otros datos. Se observó algunas historias incompletas relativas al $\mathrm{RN}$ o la madre.

Los datos se ingresaron a una base de datos utilizando el software SPSS v. 13 y epidat 3.1 previo control de calidad. Se calculó promedios y su DE, intervalos de confianza al $95 \%$ de la tasa de incidencia de los DTN a través del test de Poisson, realizándose un análisis univariado, y la comparación entre las tasas de incidencia de DTN según los diferentes años se realizó mediante una prueba de Prais Winsten.

\section{RESULTADOS}

Se encontró $128 \mathrm{RN}$ con DTN de madres cuyas edades fluctuaron entre los 14 y 44 años de edad, siendo el promedio de 26,8 años y su DE de 6,7 años. El 89,1\% (114/128), fue madres menores de 37 años; el 16,4\% (21/128) adolescentes y el 83,6\% (107/128) adultas. Con respecto a los controles prenatales $(\mathrm{CPN})$, sólo 121 gestantes tuvieron esta información registrada en la historia clínica; del total de gestantes con CPN, $62,2 \%$ tuvo cuatro a más controles prenatales $(61 / 98)$ y $37,8 \%(37 / 98)$ tuvieron menos de cuatro CPN. De un total de 51 historias clínicas con información completa acerca del antecedente de hijos con algún DTN, ninguna tuvo DTN previo y sólo en una se reportó historia familiar de DTN.

El $44,3 \%$ RN con DTN nació de parto eutócico; su edad gestacional fluctuó entre 22 y 41 semanas, siendo la edad promedio 36,2 semanas y su DE +/- 4,0 semanas; el mayor porcentaje $(66,9 \%)$ de los RN nacieron entre las 37 y 41 semanas; $54,7 \%$ fueron mujeres y el resto varones. El apgar al minuto varió entre 0 y 9 puntos; el apgar al minuto de 8 puntos fue el más frecuente y correspondió al 34,4\% (42/122); el 47,5\% (58/122) de los RN tuvieron un apgar igual o menor a 6 puntos. El apgar a los 5 minutos tuvo un rango entre 0 y 9 puntos; el $68,1 \%(81 / 119)$ de los $\mathrm{RN}$ con DTN tuvieron un apgar a los 5 minutos de 6 ó más.

Los casos incidentes de DTN fluctuaron entre 18 y 32 en todo el período de estudio; en el año 2002 se tuvo el menor número de casos incidentes $(18 / 128)$, mientras que el mayor número (32/128) se registró en el año 2003. El promedio de la tasa global de incidencia (TIG) hospitalaria de DTN en los años de estudio 2001-2005 fue de 13,6 por 10,000 RN (IC: $11,38 ; 16,21)$. Se observa un pico a mitad de período que luego baja para mantenerse estable en los 2 últimos años de estudio 2004 y 2005; se observa también una diferencia de 2 puntos porcentuales entre la TIG de 11,7 x 10000 del primer año y la TIG de 13,8 x 10000 del último año de estudio. El análisis de la evolución de las tasas de incidencia de DTN a lo largo de los años 2001 al 2005 muestra una tendencia ascendente no significativa $(p=0,951)$. En la Tabla 1 se presentan las frecuencias anuales del total de casos incidentes de DTN y sus respectivas tasas de incidencia e IC al 95\% y en la Figura 1 se aprecia con detalle la tendencia de la TIG de los 5 años estudiados.

La espina bífida fue el DTN predominante, alcanzando el 60,9\% (78/128) de los DTN, seguido de la anencefalia con el 37,5\% (48/128), mientras que el encefalocele se presentó en sólo el 1,5\% (2/128). La forma de espina bífida más frecuente fue el mielomeningocele con $88,5 \%$ $(69 / 78)$ y también con la más alta tasa de incidencia con 7,4 x 10000 (IC: 5,72;9,30). En espina bífida, hubo $84,6 \%(66 / 78)$ casos de madres adultas y 15,4\% (12/78) de madres adolescentes, mientras que para anencefalia, hubo $81,3 \%(39 / 48)$ adultas y $18,9 \%(10 / 53)$ adolescentes; los dos casos de encefalocele correspondieron a gestantes adultas.

\section{DISCUSIÓN}

Siendo uno de los objetivos de la ley de fortificación de la harina de trigo en el Perú prevenir los defectos del tubo neural, no deja de ser llamativa la falta de estudios de base necesarios como la incidencia de los DTN, los niveles de folatos en suero y en células en la 
población peruana, particularmente en las gestantes. Esta desinformación antes de la promulgación de la referida ley fue comunicada en su oportunidad por Sanabria y Tarqui ${ }^{16}$.

Por otro lado, la TIG de DTN encontrada fue inferior al rango reportado en la Eclamp Chile y el resto de la Eclamp ${ }^{7,8}$ de 17,0 por 10.000 (IC:15,79;18,25) y de 14,9 por 10,000 (IC: 14,$47 ; 15,27)$, respectivamente. Las tasas de incidencia entre los resultados de este estudio y lo reportado en Chile presentan diferencias estadísticamente significativas a juzgar por los intervalos de confianza al 95\%. Sin embargo, la tasa de DTN encontrada es mayor a la hallada en países europeos como España así como a la tasa estimada por la OMS a inicios de la década de los 80 de 10 por 10.000 nacidos vivos ${ }^{17}$.

Igualmente, nuestros hallazgos son mayores a lo encontrado en Costa Rica en la etapa prefortificación de 9,7 casos de DTN por 10.000 RN . Si comparamos las tasas de incidencia de DTN global (TIG) del año 2001 y la del año 2005, aparentemente habría una tendencia de la incidencia al incremento; sin embargo, al aplicar el test de Prais Winsten se verifica que no es significativa $(0,951)$. Por otro lado, se observa un notable incremento en la tasa de incidencia de DTN en el año 2003, situación que podría explicarse por las siguientes razones: primero, el INMP empieza a restringir la atención de los embarazos de alto riesgo y se convierte en un centro de referencia no sólo de Lima sino también de provincias $\mathrm{y}$, segundo, adquiere mayor importancia el Registro Informático Perinatal, por lo que se capacita y sensibiliza a los profesionales de la salud para que se consigne el tipo de malformación congénita en la historia clínica, evitándose el subregistro o los diagnósticos ambiguos de "malformaciones congénitas". Cabe precisar que el INMP atiende embarazos de alto riesgo que en su gran mayoría habitan en la zona urbana de Lima donde la dieta de la mujer embarazada puede jugar un rol importante -v.g. razones económicas- que a diferencia de los otros estudios de la Eclamp ${ }^{7}$, no se incluye hospitales de provincias. Otra razón para establecer diferencias entre nuestros hallazgos y la incidencia de DTN en otros países podría ser una distribución diferente de polimorfismos de la enzima metilentetrahidrofolato reductasa (Mthfr) en las poblaciones o etnias; se han descrito marcadores genéticos en la población chilena que podría significar la existencia de poblaciones con mayor riesgo de $\mathrm{DTN}^{18}$ por necesidad de mayor cantidad de folatos. Finalmente, otra razón para justificar diferencias entre las TIG de DTN puede ser la no inclusión de los mortinatos, de modo que registrar sólo los RN vivos puede significar dejar de detectar un número importante de DTN.

Antes de la fortificación en los Estados Unidos de Norteamérica se encontró una tasa de mielomeningocele de 4,4 a 4,6 casos por 10.000 nacidos vivos ${ }^{19}$. Esta cifra disminuyó hasta 3,2 casos por 10.000 nacidos en $1992^{20}$ después de la fortificación. Estudios en México muchos años antes de la fortificación revelaron una incidencia de mielomeningocele de $12 \mathrm{x}$ $10.000 \mathrm{RN}^{21}$.

La tasa de incidencia de mielomeningocele $(7,4 \times 10.000 \mathrm{RN})$ es mayor comparado con los países desarrollados como Estados Unidos de Norteamérica y es menor si se compara con México. En general, en el Perú son escasas las estadísticas al respecto y sólo se encontró una publicación del Instituto de Salud del Niño que refiere una incidencia hospitalaria de 31 por 10.000 egresos hospitalarios al año ${ }^{22}$.

La anencefalia fue el tipo de DTN más frecuente después de la espina bífida y se caracteriza por la discusión que desencadena desde el punto de vista ético y legal ${ }^{23}$. La tasa de anencefalia fue 5,1 x 10.000 nacidos vivos, cifra que es menor que la reportada en México $(15,8 \text { por } 10.000 \mathrm{NV})^{24}$ y la ECLAMP-Chile durante la etapa prefortificación 1969-1999 $(7,16$ por 10.000 (IC $95 \%: 6,37 ; 7,98))^{7}$ y de la ECLAMP del resto de países $(6,06$ por 10000 (IC:5,83;6,34)).

De acuerdo a los resultados, la anencefalia, la espina bífida y sus diversas formas tienen una menor tasa de incidencia en el INMP en comparación con los otros países de Latinoamérica en la etapa prefortificación. Los resultados son concordantes con lo referido a 


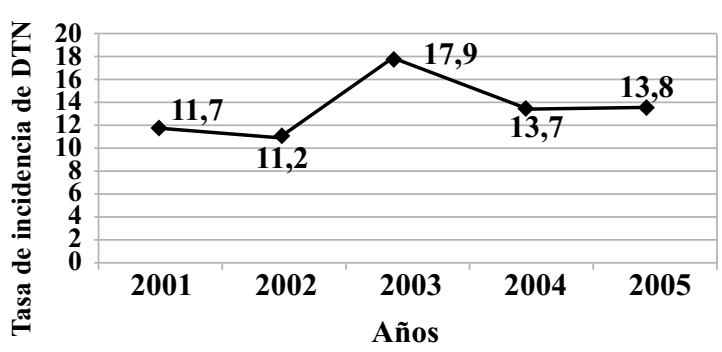

Figura 1.Tendencia de la incidencia de DTN x 10,000 (2001-2005) Estudio en el INMP de Lima, Perú.

inicios de la década de los 70 s por Cook y col. ${ }^{25}$. César Reynafarge observó cierta disminución del ácido fólico en todos los países excepto en el Perú y particularmente en las embarazadas; ellos trataron de explicar sus observaciones debido a una mayor ingesta de plátano por ser la fruta más barata, luego la lechuga y las espinacas que fueron los alimentos que más consumían en ese tiempo según la $\mathrm{ENCA}^{26}$; pero la explicación más clara se le atribuyó a la carne de pescado sin cocinar (ceviche) que se caracteriza porque contiene una apreciable cantidad de ácido fólico antes de ser consumido, aparte del ácido folínico del limón con el que se prepara.

Vieira y Castillo $^{27}$ en un estudio de metanálisis, encontraron que el riesgo de presentar alguna malformación congénita se incrementa con la edad. Estos resultados pueden considerarse parcialmente discordantes con los hallazgos de nuestro estudio que encontró que el riesgo de anencefalia y espina bífida fue mayor en gestantes mayores a 40 años - espina bífida también frecuente en adolescentes menores de 20 años-. Es importante puntualizar que los DTN fueron más frecuentes en los RN mujeres que en los hombres; siendo éstos resultados coincidentes con lo encontrado por Rodríguez y Col en México ${ }^{28}$ con 56,3\% mujeres y $43,8 \%$ hombres $^{28}$, así como por por Xiao y $\mathrm{Col}^{29}$ quienes también encontraron que las niñas eran las mas afectadas con DTN.

Dado que los DTN tienen una de las tasas de incidencia más elevadas de todas las malformaciones congénitas, el enfoque preventivo con ácido fólico en las gestantes es de carácter normativo. Las mujeres necesitan consumir ácido fólico un mes antes de embarazarse y durante las primeras semanas de desarrollo del feto. Si la mujer tiene suficiente ácido fólico en el cuerpo antes del embarazo, podrá prevenir los DTN. Sólo de esta manera la suplementación periconcepcional con ácido fólico reducirá en $72 \%$ la incidencia de DTN con lo que quedará una vez más demostrado su impacto; al respecto, una revisión de la Biblioteca Cochrane ${ }^{30}$ encontró en 4 estudios aleatorizados un riesgo relativo 0,28 (IC: 0,13 ; 0,58 ) protector del ácido fólico en relación a los DTN.

Finalmente, se hace imperativo conocer la TIG de DTN y sus tipos durante los siguientes años post fortificación para demostrar el verdadero impacto de haber fortificado la harina de trigo con ácido fólico y otros micronutrientes en el Perú. Se espera una reducción de la tendencia de la TIG de DTN en el primer quinquenio postfortificación al compararlo con el último quinquenio prefortificación. Se requiere mayor investigación en esta área de la epidemiología nutricional en el Perú y sus regiones.

\section{CONCLUSIONES}

- El promedio de la TGI de DTN en el período 2001-2005 previo a la fortificación de la harina de trigo con ácido fólico fue 13,6 por 10,000 RN (IC: 11,38;16,21).

- La tendencia de la incidencia de DTN en el INMP antes de la fortificación de la harina con ácido fólico fue ascendente pero no significativa.

- El mielomenigocele tuvo la más alta tasa de incidencia en relación a los otros tipos de DTN.

\section{RECOMENDACIONES}

- Promover la utilización de alimentos en base a la harina fortificada con ácido fólico así como el uso diario de suplementos de ácido fólico ${ }^{31}$ en todas las mujeres en edad fértil y con mayor razón si planifica un embarazo.

- El Estado Peruano debe hacer una evaluación 
del impacto de la fortificación de la harina de trigo con ácido fólico y otros micronutrientes.

Estudio presentado en la XXVII Jornadas Chilenas de Salud Pública, Escuela de Salud Pública de Chile. Santiago, Chile, 1 y 2 de octubre 2008.

\section{REFERENCIAS}

1. WALSS RR, REYES GA, ACOSTA CA, MURRA RJ, RODRÍGUEZ RE. Epidemiología de los defectos congénitos del tubo neural en la ciudad de Toncón Coahuila. Rev Mex IMSS 1990; 28:265-8.

2. GODFREY P, OAKLEY JR. Frequency of human congenital malformation. Clin Perinatol 1986; 13(3):545-54.

3. ABRAMSKY L, BOTTING B, CHAPPLE J, STONE D. Has advice on periconcepcional folate supplementation reduced neural tube defect. Lancet. 1999; 354: 998-9.

4. SEVER LE. Epidemiological aspects of neural tube defects. In: Crandall BF and Brazier MAB (eds) "Prevention of Neural Tube Defects" London: Academic Press, 1978, pp 75-89.

5. BERRY RJ, LI Z, ERICKSON JD, LI S, MOORE CA, WANG H, MULINARE J, ZHAO P, WONG LY, GINDLER J, HONG SX, CORREA A. Prevention of neural tube defects with folic acid in China (ChinaU.S. Collaborative Project for Neural Tube Defects) N. Engl. J. Med. 1999;341:1485-90.

6. RAMIREZ-ESPITIA JA, BENAVIDES FG, LACASAÑA-NAVARRO M, MARTÍNEZ JM, GARCÍA AM, BENACH J. Mortalidad por defectos del tubo neural en México, 1980-1997. Salud Publica Mex 2003;45:356-364.

7. NAZER J, LÓPEZ CAMELO J, CASTILLA EE. Estudio de 30 años de vigilancia epidemiológica de defectos de tubo neural en Chile y en Latino América. Rev Méd Chile 2001; 129: 531-9.

8. Cortes F, MELLADO C, HERTRAMPF E, ET ISAZA C, MARTINA D, ESTUPIÑÁN J, et al. Prevalencia de malformaciones congénitas diagnosticadas en las primeras 24 horas de vida. Colombia Médica 1989; 20 (4): $108-112$.

9. CORTÉS F, MELLADO C, HERTRAMPF E; et al. Frecuencia de los defectos de cierre del tubo neural en las maternidades públicas de Santiago durante el año 1999. Rev Med Chil 2001 Mar; 129(3):277-84.

10. INSTITUTO COSTARRICENSE DE INVESTIGACIÓN Y ENSEÑANZA EN NUTRICIÓN Y SALUD (Inciensa). Prevalencia de enfermedades congénitas por provincia y cantón. Centro de Registro de Enfermedades Congénitas (CREC). Costa Rica: INCIENSA, 1987-2000.

11. LOCKSMITH GJ, DUFF P. Preventing neural tube defects: The importance of periconceptional folic acid supplements. Obstetrics \& Gynecology 1998; 91: 1027-33.

12. NATIONAL RESEARCH COUNCIL. Recommended dietary allowances / Subcommittee on the Tenth Edition of the RDAs, Food and Nutrition Board, Commission on Life Sciences, National Research Council. Washington, DC: National Academy Press; 1989.

13. INTERNATIONAL CENTRE FOR BIRTH DEFECTS. Congenital malformations worldwide: A report from the International Clearinghouse for Birth Defects Monitoring Systems. Amsterda. Edit. Elsevier, 1991. p. 41-51.

14. HERTRAMPF E, CORTES F. Folic Acid fortification of wheat flour: Chile. Nutr. Rev. 2004 Jun:62(6 PT2):S44-8.L

15. MEDICAL RESEARCH COUNCIL VITAMIN STUDY RESEARCH GROUP. Prevention of neural tube defects: results of the Medical Research Council Vitamin Study. Lancet 1991;338:131-137.

16. SANABRIA H, TARQUI C. Fundamentos para la fortificación de la harina de trigo con micronutrientes en el Perú. An. Fac. Med. 2007, 68(2):185-192.

17. SANCHOS-CALVO A, MARTÍNEZ-FRÍAS ML. Estudio clínico epidemiológico de los defectos del tubo neural clasificados por los cincos puntos del mismo. An Esp Pediatr 2001;54:165-73.

18. NITSCHE F., ALLIENDE A., SANTOS L. et al. Frecuencia del polimorfismo C677T de la 5, 10metilentetrahidrofolato reductasa (MTHFR) en mujeres chilenas madres de afectados con espina bífida y en controles normales. Rev. méd. Chile, dic. 2003, vol.131, no.12, p.1399-1404. ISSN 0034-9887.

19. LARY JM, Edmonds LD. Prevalence of spina bifida at birth: United States, 1983 - 1990: a comparison of two surveillance systems. MMWR Morb Mortal Wkly Rep. 1996;45:15-26.

20. SHURTLEFT DB, LEMIRE RJ. Epidemiology, etiologic, factors, and prenatal diagnosis of open spinal dysrafism. In: Pang D (Ed): Spinal dysraphism. Neurosurg Clin North Am. 1995;6(2):183-193.

21. CANÚN-SERRANO S, ZAFRA-DE LA ROSA G. Detección de malformaciones congénitas externas. Bol Med Hosp Inf Mex 1984; 41: 21.

22. OSORIO F. Mielomeningocele e incidencia de hidrocefalia dependiente de derivaciones de LCR en niños peruanos. Trabajo de Investigación para optar el Titulo de Especialista en Neurocirugía. Facultad de Medicina de San Fernando, Unidad de Postgrado, UNMSM. 2001

23. DINIZ D. Aborto y no viabilidad fetal: debate en Brasil. Cad. Saúde Pub. Marzo - Abril 2005; 634-639.

24. JIMÉNEZ-BALDERAS E, SALAMANCA-GÓMEZ $\mathrm{F}$, MARTÍNEZ-APAC S, BRACHO-SOLÍS M. Estudio de malformaciones congénitas en 105825 nacimientos consecutivos. Bol Med Hosp Infant Mex 1985;42:744-748.

25. COOK JD, GUTNISKY A, JAMBRA M, LABARDINI MI, LAYRISSE M, LINARES J. ET 
AL. Nutricional deficiency and anemia in Latin America, a collaborative study. Blood 1971; 38:591603.

26. MINISTERIO DE ECONOMÍA Y FINANZAS. Ministerio de Alimentación. Dirección General de Informática y Estadística. Encuesta de Consumo de Alimentos (ENCA). Lima, Perú 1976.

27. VIEIRA A, CASTILLO S. Edad materna y defectos del tubo neural: evidencia para un efecto mayor en espina bífida que anencefalia. Rev Méd Chile 2005; 133: 62-70.

28. RODRÍGUEZ G, URBINAA, MUÑO M, PIMENTEL L, RIPOL U. Prevalencia de defectos del tubo neural en el sur de Veracruz. Bol. Med. Hosp. Infant Mex. 55 (5), Mayo 1998. 257-261.

29. XIAO KZ. Epidemiology of neural tube defects in China. Chung Hua I Hsueh Tsa Chih 1989; 69(4):189-191.
30. KRAMER MS. Nutritional Advice in Pregnancy [Base de datos en Internet]. England: Cochrane Database Syst Rev, 1996 [citado 15 de agosto 2008]. Disponible e n: http://web.squ.edu.o m/ medLib/MED_CD/E_CDs/health\%20development/html/ clients/cochrane/ab000149.htm

31. Lumley J, Watson L, Watson M, Bower C. Periconceptional supplementation with folate and/or multivitamins for preventing neural tube defects [Base de datos en Internet]. England: Cochrane Database Syst Rev 2001 [citado 20 de julio 2008]. Disponible en: http://web.squ.edu.om/medLib/MED_CD/E_CDs/health\%20development $/ \mathrm{html} /$ clients/cochrane/ab001056.htm

Recepción: 19 abril 2009 Aprobación: 3 septiembre 2009

Usted puede comentar éste y otros artículos publicados en la Revista Chilena de Salud Pública, enviando un correo electrónico a revistasp@med.uchile.cl 